\title{
Research and Application of Speed Control System of Urban Rail
}

\section{Transit Train}

\author{
Yang Li-bo
}

\author{
Guangdong University of Science and Technology, Dongguan, Guangdong, China
}

\begin{abstract}
Automatic train control system (ATC) is the biggest safety system in the urban rail transit train project, which plays the role of controlling the safe operation of the train and improving operation efficiency. In this paper, the train speed control scheme is putting forward through investigation, design, testing, and analysis, of the system, and the method of improvement allocation and other engineering methods are used in the design. The feasibility of this method is also demonstrated in this paper. The results showes that the automatic speed control of train can realize the automatic driving of the train, so as to reduce the manual workload, which is also an effective way that can a reduce the phenomenon of over-speed driving caused by human error.
\end{abstract}

Keywords: Speed control, Distance and speed curve, Braking

\section{Introduction}

From the perspective of train speed control mode, the train speed control can range from the manual control without equipment train, to the on-board computer aided control, ultimately to the realization of the automatic control of train's automatic operation. The train speed control equipment (ATP, ATO) can get the necessary information through the ground communication function of train make the vehicle, including the goal, speed and train operation adjustment instruction, which can be including target, speed and adjusting order of train operation etc.. How to apply it to the vehicle control? It depends on automatic train protection, automatic driving and other main functions as well as the other auxiliary functions[1]. The train control system can realize the automatic control through the internal operation of the system, the dialogue between man and machine as well as the interface of the train, so as to realize the automatic control on operating speed[2-3], as well as emergency braking control. The train speed control methods can be divided into the control on the step speed and the control on distance of the target. In this paper, the purpose as well as the method of the speed control is analyzed, calculating the dynamic adjustment factors of the speed. There are many factors that can affect the speed control, such as the input of the external and system itself[4]. Parameter selection, operation strategy as well as interference factors and so on, thus we should analyze them and adjust the countermeasures.

\section{Comparative Analysis on Different Modes of Train Speed Control}

The equipment calculation and information processing capabilities of the equipment train can be enhanced, further application of measuring speed and target distance information can 
also be used in the dynamic calculation of safe speed and advisable running speed, which can provide better auxiliary driving function, so as to achieve manual and automatic driving mode of safety protection. The train can be calculated under the safety speed protection with smooth optimization speed so as to realize the automatic driving through the train components as well as the interface of the train.

From the perspective of train speed control curve, the train automatic speed control can be divided into two kinds. The shape of the curve is as follows: one type is the ladder - shaped speed curve, which is according to the section speed control, the other type is "one section" smooth speed curve, which is according to the target distance and speed control.

\section{Train Interval Analysis}

Urban rail transit line can be divided into uplink line, downlink line, necessary auxiliary line and parking lot, vehicle can keep on cycle operating between uplink line and downlink line, which also can return at both ends (in the middle) reentry, so as to form the whole line ring road running. Usually trains keep on tracking operation in a fixed order. Because the departure density is large, the distance between trains is shorter, the tracking operation of the train can bring out lots of problems such as speed control, parking, troubleshooting and recovery order restrictions and delays, therefore, the line running order is more prominent in the the relationship of the tracking operation of the train.

The trains are running in order, it can form a close tracking system through the design, under the premise of carrying out the performance of the system, the interval between trains can be defined as the tracking interval. Taking a certain point of the line as the observing point, the time of the first train's arriving or leaving the train to the time of the next train's arriving or leaving the point can be called as the interval time, which can also be called as the tracking time interval. They are distance attributes and time attributes of the tracking relationship.

The train interval cannot be compressed and the minimum distance is safe. The train should be kept at a safe distance from the vehicle to maintain a safe speed so as to ensure full stop without conflict. Train location safety following the principle of occlusion. When the train is closed to the front and rear of the train, the close distance between trains is defined as the tracking interval under the condition of the system performance[5]. At a certain point of the line, the time when the first train arrived / left the train to the next train to arrive / leave the point is the interval time, also called the tracking time interval. They are the distance attributes and time attributes of the tracking relationship.

This kind of occlusion usually adopts the method of space interval, which is used to allow only one train to be occupied in an interval or partition, which is a technical method that train is not allowed to enter the front section, which is considered to be a non safe section. It can be divided into fixed block, quasi moving block and moving block.

\section{Section Control}

The trains can run according to the section control mode, calculating the distance and speed. The trains can allow the ladder shaped limit speed in accordance with the change of the changes if the section. The speed of the train approaching the parking point has been segmented controlled, and the speed can be adjusted according to the level of the idle interval. Fig. 1 shows the ladder shaped speed curve (see Fig1). 


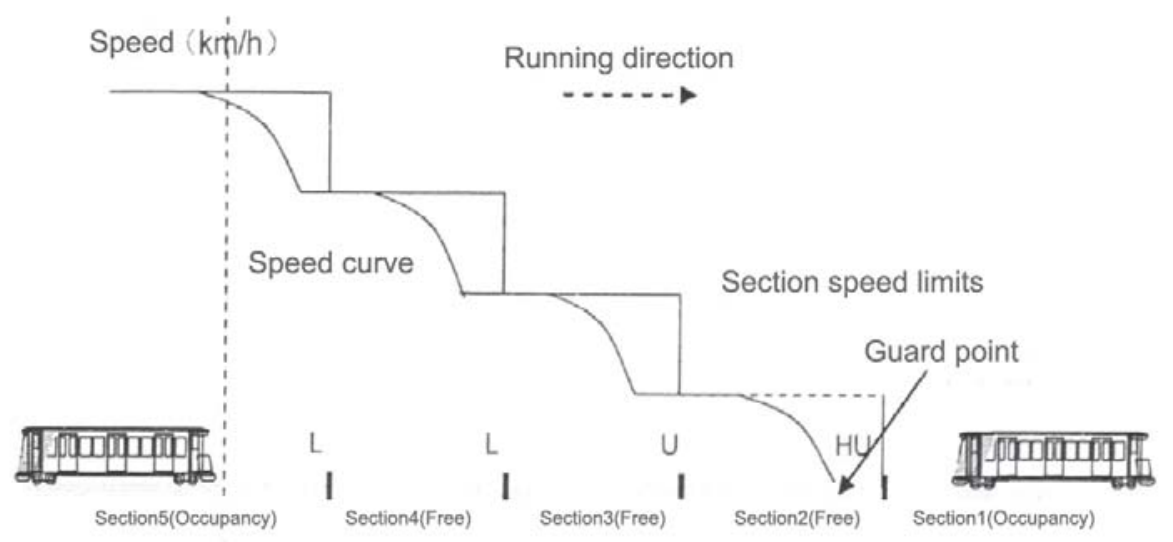

Fig. 1 Schematic Diagram of Step Speed Curve

Section control is suitable for the fixed block system. The trains can track and follow each other according to the principle of the corresponding occlusion (the conditions for the separation of different trains). A fixed block train can be divided by a predetermined number of tracks, once the interval is increased it can allow the train to travel at a higher speed. The speed of the train is controlled by the inlet speed control and the outlet speed control. The entrance speed control mode refers to the adjustment speed of the train in the entry section, trains can brake and stop in the rear section of the red band, the segment length is set to ensure the train from the dangerous point in front of the limited distance parking. The exist speed control mode refers to the train in the left section that can adjust the speed into the target speed, thus trains can run within the red zone and park beyond the segmentation point, this section can be set as the safe zone. The minimum safety interval of the train is fixed through the precalculation.

\section{Calculation of Distance Speed Curve}

The on-board subsystem can be used to calculate the safety speed monitoring curve, it can provide the recommended driving speed under the safe speed and set the alarming line.

It can form an expected speed curve that can not be beyond the emergency brake at each safe point. While the emergency intervention curve is under this line, which can make ATP carry out emergency braking, when it doesn't reach the curve.

Recommended speed curve is under the emergency intervention curve, it can set a certain margin, which can be suitable for driver's operation, such as $5 \mathrm{kmlh}$. The recommended speed is the more secure and smooth curve, with the enough running time, the loose driving curve can be adopted. With ATO mode, the needs of vehicle traction and braking through the servo control circuit ton operate in accordance with the recommended speed curve calculation. The recommended speed curve can park at the non-safe operation point, while the operation parking point is located in front of the security point, the distance of the sate interval can prevent the safety of a variety of the most unfavorable circumstances against the conflict of the parking point.

Set the alarming line between the recommended speed curve and the emergency intervention curve. If the line is violated, the sound alarm will be generated. Between the recommended speed curve and the alarming line, it can set a range within the recommended speed, which can make the driver drive in a certain speed range, meanwhile the distance 
between the alarming line and the emergency intervention line can offer the driver enough time to make reaction.

Calculation of on-board vehicle speed diagram can be shown as follows. The process of the on-board vehicle speed calculation and adjustment can be including instructions, conditions, measurement information acquisition, allowable speed and recommended speed curve, speed comparison and control output (see Fig.2).

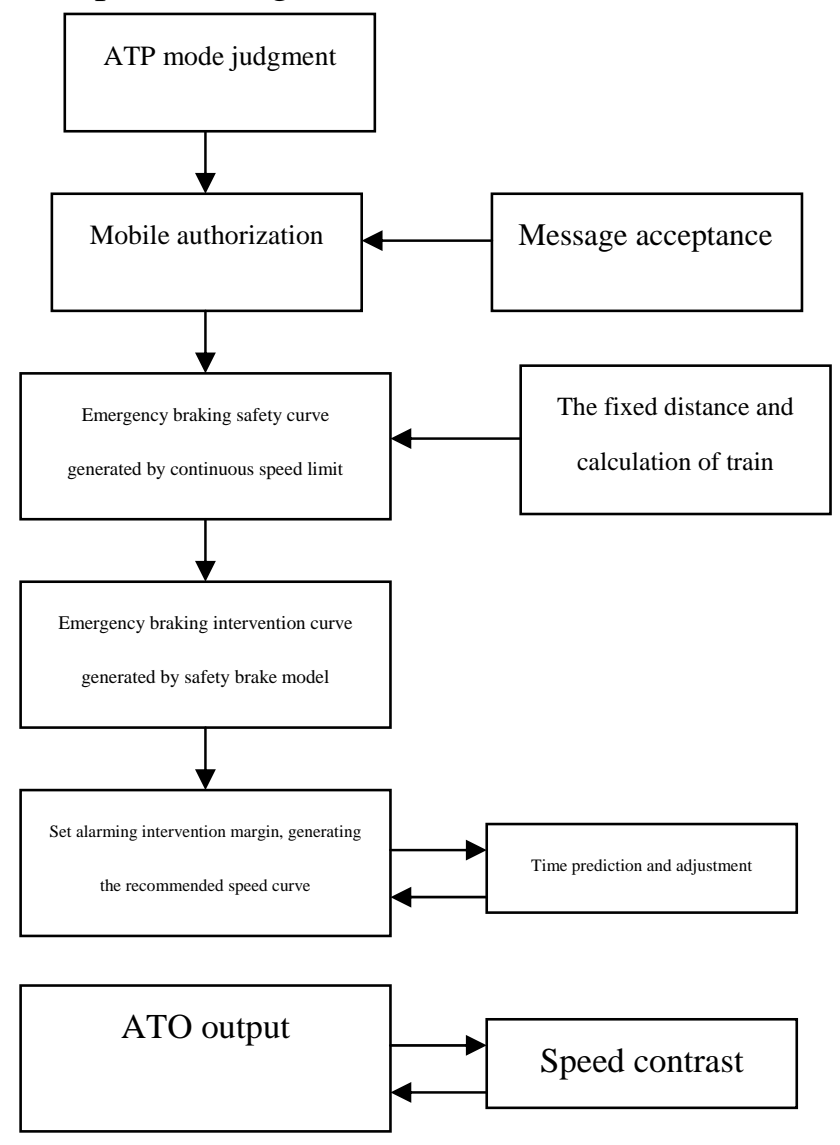

Fig. 2 Diagram of Speed Calculation

\section{The Determination of Safe Braking Model}

The calculation of control speed curve is based on safety brake model. To determine the safety brake model, you need to define four conditions, which can describe the occurrence of the target rate from the speeding up until the tight activation, so as to ensure the command can achieve the target speed with the actual effect when it is under the most adverse circumstances.

1) The response time of the emergency braking (which includes the reaction time of the on-board subsystem, the traction is considered to achieve to its maximum)

2) The reaction time to lift the train traction (the traction is considered to achieve to its maximum )

3) To establish the guaranteed rate of the reaction time (since the strength of the system is not sufficient to make the train really braked, assuming that the train is running in an idle state)

4) The time of tightening

It can be assured according to the safety brake model, changing the speed difference of the speed control curve, so as to determine the limitation of safe distance respectively. The determination of the safe distance is related to the design of the layout of the line. It can be 
seen that the vehicle performance parameters, slope as well as the set of the system response time can be directly related to the results of speed control.

\section{Conclusion}

With the improvement of the operational performance of the on-board vehicle configuration equipment, the on-board vehicle part is upgraded from the monitoring device to the monitoring safety device, its function changed from the over-speed protection to the full automatic speed control. The innovation of the on-board train speed control mode can directly improve the train control driving mode, combined with real-time distance information and train line information, it can realize intelligent train driving, which can also improve train tracking block mode as well as the line capacity. Moreover, it is also the foundation of the other functions, such as the interval automatic adjustment function. Compatible with the moving block, the on-board vehicle speed control method can set aside the method of the speed step control, the train speed can adopt target distance control mode (which can also be called as continuous speed control), according to target distance, target speed and the performance of the train itself to determine the train braking curve to adopt the method of braking.

The automatic speed control of train can realize the automatic driving of the train, so as to reduce the manual workload, which is also an effective way that can a reduce the phenomenon of over-speed driving caused by human error. Automatic speed control can reduce the speed fluctuation of manual control, which van effectively improve the operation speed and save the travel time. At the same time, the automatic operation speed is helpful to the smooth operation, which can reduce the acceleration and deceleration switch, so as to improve the comfortable sense. With the improvement of automation degree, the system has increased the station precise parking, door operation, timing and synchronization function of screen doors as well as some other functions.

\section{Reference}

[1] Dong, Hairong, et al. "Automatic train control system development and simulation for high-speed railways." IEEE circuits and systems magazine10.2 (2010): 6-18.

[2] Slane, Francis L., and Truman L. Allison. "Fault monitoring and reporting system for trains." U.S. Patent No. 4,041,470. 9 Aug. 1977.

[3] Xueyan, Chen, and Sun Shouchuang. "Application of Improved Wavelet Neural Network in Fault Diagnosis." Journal of Applied Science and Engineering Innovation 2.5 (2015): 146-149.

[4] Hong-guo, Shi, Peng Qi-yuan, and Guo Han-ying. "Traction calculation model of urban mass transit [J]." Journal of Traffic and Transportation Engineering 4 (2005): 004.

[5] Zhou, Quan, Chao Liu, and Jianjun Xu. "The influence of IMOOC" on the cultivation of the students in the electrical engineering." Journal of Applied Science and Engineering Innovation 2.7 (2015): 291-293. 